\title{
Viscoelastic testing: It's not the measurement, it's what you do with it!
}

\author{
Roman M. Sniecinski, MD, MSc
}

Received: 17 August 2018/Accepted: 18 August 2018/Published online: 6 September 2018

(C) Canadian Anesthesiologists' Society 2018

"Measure what is measurable, and make measurable what is not so." Galileo Galilei

Anesthesiologists love to measure things. Heart rate, blood pressure, and pulse oximetry values all provide information that aids in directing anesthetic care. In fact, these measurements are necessary to provide a safe anesthetic. Laboratory measurements also play a role in guiding therapy, albeit without the instant gratification of vital sign changes. For example, most practitioners would feel uncomfortable administering insulin to a patient without the ability to measure a blood glucose level at some point. Transfusion of blood products is a more complex issue, but one that also relies heavily on measurement—or at least it should.

The use of viscoelastic hemostatic testing (VHT) in surgical patients has exploded over the past decade, in part because of its ability to simultaneously measure several components of coagulation. No less than a half dozen different perioperative Societies have published guidelines recommending its use for directing the transfusion of hemostatic blood products. ${ }^{1-4}$ Yet, questions still remain about the efficacy of this testing modality, including how we act upon the information it provides. In this issue of the Journal, Lodewyks et al. present a systematic review and meta-analysis of VHT in cardiac surgical patients. ${ }^{5}$ Using 11 different randomized-controlled trials involving over 8,000 patients, the authors found no reduction in the proportion of patients exposed to allogenic blood products. They conclude that "the benefits associated with viscoelastic testing in cardiac surgery patients are insufficiently robust to recommend routine

R. M. Sniecinski, MD, MSc ( $\square)$

Department of Anesthesiology, Emory University School of Medicine, 1364 Clifton Rd, NE, Atlanta, GA 30322, USA

e-mail: rsnieci@emory.edu implementation". 5 This statement is likely to raise many readers' eyebrows and deserves further discussion to place it in the proper context.

As pointed out by Lodewyks et al., two similar metaanalyses on this subject have been recently performed. ${ }^{6,7}$ While these publications did not share the same primary outcome (see Table 1), it is important to note that all considered exposure to platelet transfusion only as a secondary outcome. Since VHT measurements can be used to directly influence administration of this blood component ( $v s$, say, red blood cells), platelet transfusion likely represents the most relevant metric to evaluate the effectiveness of VHT. Arguably, it is at least as pertinent as any of the other proposed primary outcomes listed in Table 1. In fact, I would submit that expecting a point-ofcare test to influence mortality (a primary end point in Lodewyks et al. $)^{5}$ is a pretty tall order! In any case, it is fascinating to note that despite assessing disparate, albeit overlapping, literature (five studies in common) for the secondary outcome of platelet exposure, all meta-analyses arrived at very similar effect estimates-using VHT lowers the risk of receiving a platelet transfusion by approximately $30 \%$. Such consistency in the face of highly heterogenous search strategies and analyses probably indicates a positive effect.

Unfortunately, a major limitation with all meta-analyses involving VHT is the lack universal transfusion thresholds. Considering the seven studies assessed for platelet administration in the Lodewyks et al. meta-analysis, ${ }^{8-14}$ there was considerable variability in the values that would trigger transfusion (Table 2). Even studies employing the same VHT modality (i.e., TEG [thromboelastography] or ROTEM [rotational thromboelastometry]) used different trigger threshold values for transfusing platelets. The problem is that there are few published data to indicate 
Table 1 Recent meta-analyses on viscoelastic hemostatic testing in cardiac surgical patients

\begin{tabular}{|c|c|c|c|}
\hline Authors (publication year) & Lodewyks et al. $^{5}$ (2018) & $\begin{array}{l}\text { Serraino \& Murphy } \\
\text { (2017) }\end{array}$ & Deppe et $a l .{ }^{6}(2016)$ \\
\hline Primary outcome & $\begin{array}{l}\text { Transfusion of any blood } \\
\text { product }\end{array}$ & All-cause mortality & $\begin{array}{l}\text { Various clinical } \\
\text { outcomes }\end{array}$ \\
\hline Relevant studies identified by the systematic review & $11 \mathrm{RCTs}$ & 15 RCTs & $\begin{array}{l}9 \text { RCTs } \\
8 \text { Observational }\end{array}$ \\
\hline $\begin{array}{l}\text { No. of patients in secondary analysis of exposure to a platelet } \\
\text { transfusion }\end{array}$ & 8,029 & $1,116^{*}$ & 6,589 \\
\hline RR of platelet transfusion $(95 \% \mathrm{CI})$ & $0.66(0.49$ to 0.90$)$ & $0.76(0.63 \text { to } 0.91)^{*}$ & $0.62(0.42$ to 0.92$)$ \\
\hline
\end{tabular}

$\mathrm{CI}=$ confidence interval; $\mathrm{RCT}=$ randomized-controlled trial; $\mathrm{RR}=$ relative risk of platelet exposure comparing those with viscoelastic hemostatic testing to controls. *Excluding the Karkouti et al. ${ }^{11}$ study; RR $=0.78$ (95\% CI, 0.66 to 0.93 ) with these data included

Table 2 Viscoelastic hemostatic testing thresholds for transfusion of platelets used in the Lodewyks et al. ${ }^{5}$ meta-analysis

\begin{tabular}{|c|c|}
\hline Component study & Threshold value for transfusion of any platelets \\
\hline Ak et $a l .{ }^{8}$ & TEG MA $<48 \mathrm{~mm}$ \\
\hline Avidan et al $^{9}$ & TEG utilized, but no transfusion threshold specified in paper \\
\hline Girdauskas et al. ${ }^{10}$ & ROTEM MCF on HEPTEM $\leq 45 \mathrm{~mm}$ \\
\hline Karkouti et al. ${ }^{11}$ & ROTEM A10 on EXTEM $<35 \mathrm{~mm}$ \\
\hline Kultufan Turan et al. ${ }^{12}$ & “Abnormal” TEG MA (<59 mm assumed) \\
\hline Shore-Lesserson et al. $^{13}$ & TEG MA $<45 \mathrm{~mm}$ \\
\hline Weber et al. ${ }^{14}$ & ROTEM A10 on EXTEM $\leq 40 \mathrm{~mm}$ \\
\hline
\end{tabular}

A10 = amplitude at ten minutes; EXTEM = tissue factor activation; HEPTEM = heparinase assay; MA = maximum amplitude; $\mathrm{MCF}=$ maximum clot firmness

TEG (Heamonetics, Braintree, MA, USA), ROTEM (Instrumentation Laboratory, Bedford, MA, USA)

the optimal trigger point. Unlike studies concerned with red cell transfusion, there has never been an attempt to classify hemostatic blood product transfusion algorithms as "restrictive" or "liberal". This is not surprising since even baseline VHT measurements in "healthy" patients are not well defined. ${ }^{15}$ Thus, a meta-analysis of VHT effectiveness reveals more about transfusion algorithms, which tend to be highly variable, than the testing modality itself.

The usefulness of measurements cannot be assessed if the actions they produce are inappropriate. Unfortunately, there are scant high-quality data to help guide the appropriate response to a given VHT measurement. Who is to say that a TEG maximum amplitude value of $45 \mathrm{~mm}$ requires a platelet transfusion, while a value of $50 \mathrm{~mm}$ does not? Is there even any clinical difference between the two values? The situation is made all the more complex because although VHT is considered to be a "global hemostatic assay", critical aspects of coagulation such as the endothelium and shear forces are not measured. While VHT parameters are certainly useful, more basic research is needed to reveal what they reflect.

All of this brings us back to the conclusion by Lodewyks et $a .^{5}$ While I agree that VHT is not ready for "routine implementation", I would emphasize the word routine. Anyone hoping to reduce blood product transfusion by purchasing a TEG or ROTEM device and blindly following an algorithm is likely to be very disappointed. Nevertheless, this should not be misinterpreted to mean that VHT should be abandoned. On the contrary, VHT information, just like a hemoglobin value, should be obtained when transfusion is being contemplated, but must also be interpreted in the context of the patient's baseline coagulation status and the ongoing clinical situation. Coagulation is wonderfully complex, which should provide anesthesiologists all the more incentive to measure it.

\section{Tests de visco-élasticité sanguine : ce n'est pas la mesure qui compte, c'est la façon dont vous l'utilisez!}

«Mesurer ce qui est mesurable et rendre mesurable ce qui ne l'est pas ». Galilée 
Les anesthésiologistes aiment mesurer. Les valeurs de la fréquence cardiaque, de la tension artérielle et de l'oxymétrie du pouls artérielle fournissent toutes des renseignements qui contribuent à l'orientation des soins anesthésiques. De fait, ces mesures sont nécessaires pour assurer une anesthésie sécuritaire. Les résultats d'analyses de laboratoire ont également leur place pour guider le traitement, quoique sans apporter la satisfaction immédiate des modifications des constantes vitales. La majorité des praticiens se sentirait mal à l'aise, par exemple, en administrant de l'insuline à un patient sans pouvoir mesurer la glycémie. La transfusion de produits sanguins et un problème plus complexe, mais en est un qui repose fortement sur des résultats de laboratoire ou qui, du moins, le devrait

L'utilisation des tests de visco-élasticité hémostatique (TVH) chez les patients chirurgicaux a littéralement explosé au cours des dix dernières années en partie grâce à la possibilité de mesurer simultanément plusieurs éléments de la coagulation. Pas moins d'une demi-douzaine de sociétés savantes différentes traitant des questions périopératoires ont publié des lignes directrices recommandant son utilisation pour guider la transfusion de produits sanguins hémostatiques. ${ }^{1-4}$ Des questions restent cependant ouvertes sur l'efficacité de cette modalité de tests, notamment sur la façon dont nous agissons en regard de l'information fournie. Dans ce numéro du Journal, Lodewyks et coll. présentent une revue systématique de la littérature et une méta-analyse des TVH chez les patients subissant une chirurgie cardiaque. ${ }^{5}$ À partir de 11 essais contrôlés randomisés différents ayant inclus plus de 8000 patients, les auteurs ont trouvé qu'il n'y avait pas de réduction du pourcentage de patients exposés à des produits sanguins allogéniques. Ils concluent donc que «les bénéfices associés aux tests de visco-élasticité chez les patients de chirurgie cardiaque ne sont pas suffisamment solides pour recommander leur implémentation dans la pratique courante $» .^{5}$ Une telle affirmation fera sans doute froncer les sourcils de nombreux lecteurs et mérite une plus large discussion pour la replacer dans son contexte.

Comme l'indiquent Lodewyks et coll., deux méta-analyses similaires sur ce sujet ont été récemment effectuées. ${ }^{6,7}$ Bien que le critère d'évaluation principale n'ait pas été le même dans ces publications (voir le Tableau 1), il est important de remarquer que toutes tenaient compte de l'exposition aux transfusions de plaquettes que comme critère d'évaluation secondaire. Dans la mesure où les résultats des TVH peuvent servir à influencer directement l'administration de cet élément sanguin (contre, par exemple, la transfusion de globules rouges), la transfusion de plaquettes est susceptible de représenter le critère mesurable le plus pertinent pour évaluer l'efficacité des TVH. Du moins peut-on dire qu'il s'agit d'un critère aussi pertinent que les autres critères d'évaluation principaux proposés qui sont énumérés dans le Tableau 1. En fait, j'aurais tendance à penser que la possibilité qu'un test au point de service puisse influencer la mortalité (un critère d'évaluation principale dans l'analyse de Lodewyks et coll. $)^{5}$ est un objectif plutôt optimiste! Dans tous les cas, il est fascinant de constater qu'en dépit d'une évaluation d'articles disparates, en dépit d'un certain chevauchement (cinq études communes) sur le critère d'évaluation secondaire de l'exposition aux plaquettes, toutes les méta-analyses sont parvenues à des estimations de l'effet très semblables : l'utilisation des TVH abaisse le risque de recevoir une transfusion de plaquettes d'environ $30 \%$. Une telle constance face à des stratégies de recherche et des analyses très hétérogènes est probablement l'indication d'un effet positif.

Malheureusement l'une des principales limites de toutes les analyses portant sur les TVH est l'absence de seuil universel de transfusion. Considérant les sept études évaluées pour l'administration de plaquettes dans la méta-analyse de Lodewyks et coll., ${ }^{8-14}$ la variabilité des valeurs déclenchant l'indication d'une transfusion est considérable (Tableau 2). Même les études utilisant la même modalité de TVH (c'est-à-dire, TEG [thromboélastographie] ou ROTEM [la thromboélastométrie rotative]) ont utilisé des valeurs seuils différentes pour les transfusions de plaquettes. Le problème est qu'on ne dispose que de peu de données publiées nous indiquant le point de déclenchement optimal. Contrairement aux études portant sur les transfusions de globules rouges, il n'y a jamais eu de tentative de classification des algorithmes de transfusion de produits sanguins hémostatiques en « restrictifs » ou « libéral ». Ceci n'est pas surprenant dans la mesure où même les valeurs de base des tests de viscosité chez les patients « en bonne santé » ne sont pas bien définies. ${ }^{15}$ Par conséquent, une méta-analyse sur l'efficacité des TVH en dit plus sur les algorithmes transfusionnels (qui tendent à être extrêmement variable) que sur la modalité du test proprement dite.

L'utilité des mesures ne peut pas être évaluée si les actions qu'elles entraînent sont inadaptées. Nous ne disposons, hélas, que de bien peu de données de grande qualité pour guider une réponse appropriée à une mesure donnée d'un TVH. Qui peut dire que la valeur maximum de l'amplitude d'un TEG de $45 \mathrm{~mm}$ requiert une transfusion plaquettes et qu'une valeur de $50 \mathrm{~mm}$ ne le justifie pas? Existe-t-il même une différence clinique entre ces deux valeurs? La situation est rendue encore plus complexe, car bien que le TVH soit considéré comme étant un « test hémostatique global », des éléments essentiels de la coagulation, tels que l'endothélium et les forces de 
Tableau 1 Méta-analyses récentes sur le test hémostatique de visco-élasticité sanguine chez les patients subissant une chirurgie cardiaque

\begin{tabular}{|c|c|c|c|}
\hline Auteurs (année de publication) & $\begin{array}{l}\text { Lodewyks } \text { et coll. }^{5} \\
\text { (2018) }\end{array}$ & $\begin{array}{l}\text { Serraino \& } \\
\text { Murphy }^{7}(2017)\end{array}$ & Deppe et coll. ${ }^{6}(2016)$ \\
\hline Critère d'évaluation principal & $\begin{array}{l}\text { Transfusion de tout } \\
\text { produit sanguin }\end{array}$ & $\begin{array}{l}\text { Mortalité toutes } \\
\text { causes }\end{array}$ & $\begin{array}{l}\text { Divers critères } \\
\text { d'évaluation cliniques }\end{array}$ \\
\hline Études pertinentes identifiées par l'analyse systématique & $11 \mathrm{ERC}$ & $15 \mathrm{ERC}$ & $\begin{array}{l}9 \text { ERC } \\
8 \text { études observationnelles }\end{array}$ \\
\hline $\begin{array}{l}\text { Nombre de patients dans l'analyse secondaire de l'exposition à une } \\
\text { transfusion de plaquettes }\end{array}$ & 8029 & $1116^{*}$ & 6589 \\
\hline RR de transfusion de plaquettes (IC à $95 \%$ ) & $0,66(0,49$ à 0,90$)$ & $0,76(0,63 \text { à } 0,91)^{*}$ & $0,62(0,42$ à 0,92$)$ \\
\hline
\end{tabular}

IC = intervalle de confiance; ERC = essais cliniques randomisés contrôlés; RR = risque relatif d'exposition aux plaquettes comparant le test hémostatique de viscosité et les contrôles. *À l'exclusion de l'étude de Karkouti et coll.; ${ }^{11} \mathrm{RR}=0,78$ (IC à $95 \%, 0,66$ à 0,93$)$ en incluant ces données

Tableau 2 Seuils des tests hémostatiques de visco-élasticité pour les transfusions de plaquettes utilisés dans la méta-analyse de Lodewyks et coll. $^{5}$

\begin{tabular}{|c|c|}
\hline Étude des composants & Valeur seuil pour une transfusion de plaquettes \\
\hline Ak et coll. ${ }^{8}$ & TEG MA $<48 \mathrm{~mm}$ \\
\hline Avidan et coll..$^{9}$ & TEG utilisé, mais aucun seuil de transfusion précisé dans l'article \\
\hline Girdauskas et coll. ${ }^{10}$ & ROTEM MCF sur HEPTEM $\leq 45 \mathrm{~mm}$ \\
\hline Karkouti et coll. ${ }^{11}$ & ROTEM A10 sur EXTEM $<35$ mm \\
\hline Kultufan Turan et coll. ${ }^{12}$ & TEG MA « anormal » (<59 mm - supposition $)$ \\
\hline Shore-Lesserson et coll $^{13}$ & TEG MA $<45 \mathrm{~mm}$ \\
\hline Weber et coll. ${ }^{14}$ & ROTEM A10 sur EXTEM $\leq 40 \mathrm{~mm}$ \\
\hline
\end{tabular}

A10 = amplitude à dix minutes; EXTEM = activation du facteur tissulaire; HEPTEM = avec héparinase; MA = amplitude maximum; MCF = fermeté maximum du caillot

TEG (Heamonetics, Braintree, MA, É-U.), ROTEM (Instrumentation Laboratory, Bedford, MA, É-U.)

cisaillement, ne sont pas mesurés. Alors que les paramètres des TVH sont certainement utiles, nous avons besoin de plus de recherche fondamentale pour interpréter ce qu'ils mesurent.

Tout ceci nous ramène à la conclusion de Lodewyks et coll..$^{5} \mathrm{Si}$ je suis d'accord sur le fait que les TVH ne sont pas prêts pour une «implémentation dans la pratique courante », j'insisterai sur le qualificatif courante. Quiconque espérant réduire les transfusions de produits sanguins en achetant un dispositif TEG ou ROTEM et en suivant aveuglément un algorithme risque une très grande déception. Cela ne doit quand même pas être mal interprété comme signifiant que les TVH devraient être abandonnés. Au contraire, l'information fournie par le TVH, au même titre que la valeur de l'hémoglobine, doit être obtenue quand on envisage une transfusion mais doit aussi être interprété dans le contexte du statut de coagulation initiale du patient et dans celui de la situation clinique en cours. La coagulation est merveilleusement complexe et cela devrait inciter d'autant plus les anesthésiologistes à la mesurer.
Conflicts of interest None declared.

Editorial responsibility This submission was handled by Dr. Steven Backman, Associate Editor, Canadian Journal of Anesthesia.

Conflit d'intérêt Aucun déclaré.

Responsabilité éditoriale Cet article a été traité par le $\mathrm{D}^{\mathrm{r}}$ Steven Backman, rédacteur adjoint, Journal canadien d'anesthésie.

\section{References}

1. Society of Thoracic Surgeons Blood Conservation Guideline Task Force; Ferraris VA, Brown JR, Despotis GJ, et al. 2011 update to the Society of Thoracic Surgeons and the Society of Cardiovascular Anesthesiologists blood conservation clinical practice guidelines. Ann Thorac Surg 2011; 91: 944-82.

2. Kozek-Langenecker SA, Ahmed AB, Afshari A, et al. Management of severe perioperative bleeding: guidelines from the European Society of Anaesthesiology: First update 2016. Eur J Anaesthesiol 2017; 34: 332-95. 
3. Task Force on Patient Blood Management for Adult Cardiac Surgery of the European Association for Cardio-Thoracic Surgery (EACTS); European Association of Cardiothoracic Anaesthesiology (EACTA); Boer C, Meesters MI, Milojevic M, et al. 2017 EACTS/EACTA guidelines on patient blood management for adult cardiac surgery. J Cardiothorac Vasc Anesth 2018; 32: 88-120.

4. Roullet $S$, de Maistre E, Ickx B, et al. Position of the French Working Group on Perioperative Haemostasis (GIHP) on viscoelastic tests: what role for which indication in bleeding situations? Anaesth Crit Care Pain Med 2018. DOI: https://doi. org/10.1016/j.accpm.2017.

5. Lodewyks C, Heinrichs J, Grocott HP, et al. Point-of-care viscoelastic hemostatic testing in cardiac surgery patients: a systematic review and meta-analysis. Can J Anesth 2018. DOI: https://doi.org/10.1007/s12630-018-1217-9.

6. Deppe AC, Weber C, Zimmermann $J$, et al. Point-of-care thromboelastography/thromboelastometry-based coagulation management in cardiac surgery: a meta-analysis of 8332 patients. J Surg Res 2016; 203: 424-33.

7. Serraino GF, Murphy GJ. Routine use of viscoelastic blood tests for diagnosis and treatment of coagulopathic bleeding in cardiac surgery: updated systematic review and meta-analysis. $\mathrm{Br} \mathrm{J}$ Anaesth 2017; 118: 823-33.

8. Ak K, Isbir CS, Tetik S, et al. Thromboelastography-based transfusion algorithm reduces blood product use after elective CABG: a prospective randomized study. J Card Surg 2009; 24 : 404-10.

9. Avidan MS, Alcock EL, Da Fonseca J, et al. Comparison of structured use of routine laboratory tests or near-patient assessment with clinical judgement in the management of bleeding after cardiac surgery. Br J Anaesth 2004; 92: 178-86.

10. Girdauskas E, Kempfert J, Kuntze $T$, et al. Thromboelastometrically guided transfusion protocol during aortic surgery with circulatory arrest: a prospective, randomized trial. J Thorac Cardiovasc Surg 2010; 140(1117-24): e2.

11. Karkouti K, Callum J, Wijeysundera DN, et al. Point-of-care hemostatic testing in cardiac surgery: a stepped-wedge clustered randomized controlled trial. Circulation 2016; 134: 1152-62.

12. Kultufan Turan S, Ayik I, Yagar S, Kazanci D, Karadeniz U, Ozgok A, Erdemli $O$. The role of rotaitonal thromboelastography on decision of blood transfusion in open heart surgery. GKD Anest Yoj Bak Dem Ders 2006; 12: 154-9. Kultufan Turan S, Aydinli B, Ayik I, et al. The role of rotational thromboelastography on decision of blood transfusion in open heart surgery; 2006; 12: 154.

13. Shore-Lesserson L, Manspeizer HE, DePerio M, Francis S, VelaCantos F, Ergin MA. Thromboelastography-guided transfusion algorithm reduces transfusions in complex cardiac surgery. Anesth Analg 1999; 88: 312-9.

14. Weber $C F$, Görlinger K, Meininger D, et al. Point-of-care testing: a prospective, randomized clinical trial of efficacy in coagulopathic cardiac surgery patients. Anesthesiology 2012; 117: 531-47.

15. Adler M, Ivic S, Bodmer DS, et al. Thromboelastometry and thrombelastography analysis under normal physiological conditions - systematic review. Transfus Med Hemother 2017; 44: 78-83. 